\title{
Field strength correlators in the instanton gas: The importance of the two-instanton contribution
}

\author{
E.-M. Ilgenfritz ${ }^{1}$, B. V. Martemyanov ${ }^{2}$, M. Müller-Preussker ${ }^{3}$ \\ ${ }^{1}$ Research Center for Nuclear Physics, Osaka University, Japan \\ ${ }^{2}$ Institute of Theoretical and Experimental Physics, Moscow, Russia \\ ${ }^{3}$ Institut für Physik, Humboldt-Universität zu Berlin, Germany
}

\begin{abstract}
The field strength correlators at zero temperature are semi-classically evaluated fitting the random instanton liquid model to lattice data for quenched $S U(3)$ lattice gauge theory. We restrict ourselves to the lowest order in an instanton density expansion necessary to explain the difference between $\mathcal{D}_{\perp}$ and $\mathcal{D}_{\|}$. In the instanton-instanton and instanton-antiinstanton contributions the Schwinger line factors - neglected in a previous analysis - are numerically taken into account in a weighted Monte Carlo evaluation. This leads to different estimates for instanton size and density. A reasonable description of the correlators within the intermediate range from $0.4 \mathrm{fm}$ to $1 \mathrm{fm}$ is obtained.
\end{abstract}

\section{INTRODUCTION}

Based on the concept of gluon field-strength correlators, a model independent, albeit systematic description of non-perturbative effects in QCD [1] 3as been worked out. Few correlation functions play an important role in the stochastic confinement model and give a detailed description of the level splitting of heavy and light $\bar{Q} Q$ bound states [4]. Relying on the analytical 
continuation from Euclidean to Minkowski space, they provide a bridge between low and high energy QCD phenomenology: they are the basic ingredients for a description of high-energy hadron and quark-(anti)quark scattering within the stochastic vacuum approach [0].

By now, numerical results are available from lattice simulations concerning the two-point field strength correlators for pure gauge theory with the gauge groups $S U(2)$ [6 8 ] and $S U(3)$ [9 12] over physical distances ranging up to $1 \mathrm{fm}$. The correlators have also been obtained near the deconfinement transition in pure $S U(3)$ gauge theory [10]. Somewhat later, this study has been extended to full QCD with four flavours of dynamical staggered quarks [11].

In most of the recent lattice computations of the gluonic field strength correlators the signal has been extracted using a cooling procedure which serves the purpose to erase short-range fluctuations on the level of a few lattice spacings, thereby redefining (renormalizing) the field strength operator on the lattice. Apart from this renormalization, the correlator is expected to be not affected by this procedure as long as the diffusive cooling radius $r \propto \sqrt{n}$ remains small compared to the distances $d$ of interest (in units of lattice spacing) for an appropriate number $n$ of cooling iterations. Presently, this prevents the knowledge of the field strength correlator at very short distances below $0.1 \mathrm{fm}$. Going to these distances requires to do such simulations on a few times finer lattices. In the finite temperature case and for QCD with dynamical fermions the correlators are presently known only for distances larger than $0.4 \mathrm{fm}$.

With cooling like this, eventual semiclassical structures underlying the vacuum of YangMills theory or full QCD can hardly be revealed from Monte Carlo lattice configurations. Nevertheless, one might try - on the basis of the lattice data for the field strength correlators - to justify and to constrain semiclassical models of vacuum structure. By definition, correlators formed by the field strengths of particular semiclassical configurations do not cover the perturbative part of what is measured on the lattice. In order to compare, one has to rely completely on the way how the lattice data are split into a (singular at short distances) lowest order perturbative part and the non-perturbative signal. This is the way how the renormalon ambiguity is presently dealt with (for a discussion see the recent talk by Di Giacomo [13]). The non-perturbative signal is usually modelled by exponential contributions to the two basic 
structure functions $\mathcal{D}$ and $\mathcal{D}_{1}$ (see below). These can be replaced by other functional forms [14], e.g. suggested within high energy scattering phenomenology [15], or provided by some specific vacuum model as in the present paper.

Instantons - localized finite action solutions of the Euclidean Yang Mills field equations are well-known examples of semiclassical configurations [16], which have been put into hadronic phenomenology in a dilute gas model in Ref. [17]. After the IR divergence had been cured in a rather ad hoc way taking interactions into account [18], the instanton liquid model has been developed by Shuryak [19] and Diakonov [20]. For reviews we refer to Refs. 21, 22]. Gradually, since instantons are now successfully identified in lattice ensembles (see the recent rapporteur talks [23,24]), the instanton vacuum accumulates direct support from lattice QCD. However, there are still systematic uncertaincies concerning the parameters of the instanton liquid emerging from lattice analyses.

Without doubt, instantons play an important role in explaining chiral symmetry breaking and other empirical facts of hadron structure. However, their contribution to the confinement property of non-Abelian gauge theory (as far as this is established on the lattice) turned out quantitatively insufficient [25,26], at least at the present level of analytically dealing with the instanton liquid model or implementing it in numerical simulation. One step beyond the current wisdom, i.e. beyond the neglect of instanton color correlations, has been attempted in Ref. [27]. The method proposed there uses the field strength correlator in a dedicated way between clusters of topological charge. However, based on the RG smoothing method, the instanton interpretation of the emerging clusters in [27] has remained uncertain. Therefore, the same type of measurement should be applied in the context of any cooling study devoted to instantons.

Relating instantons to the confinement issue, alternatively to the $\bar{Q} Q$ force, the property of monopole percolation in a multiinstanton system [25,28] or the effect of instantons on the vacuum structure as summarized in the field strength correlators can be investigated. Earlier studies of field strength correlators based on the instanton liquid model can be found in Refs. [29 31]. Here, there are two lines of thought. One [30,32 relies on the one-instanton approximation and concentrates on the modification of the single instanton approximation (and 
the instanton solution by itself) due to the interaction with the vacuum medium surrounding it. Our approach tries to separate the contributions of one (anti)instanton from contributions when the field strength comes from the non-linear superposition of two (anti)instantons. Correspondingly, different results can be found in Refs. [29 31].

As far as the one-instanton contribution is concerned, we have nothing to add to our previous paper [31]. Concerning the two-instanton contributions, we had followed the current wisdom and neglected eventual color correlations in the instanton liquid. This is not a bad approximation for zero temperature quenched QCD, and we keep this approximation also now. However, our results in the previous paper were obtained omitting the Schwinger-line phase factors in dealing with the two-instanton contributions. Some (rough) arguments led us to expect a negligible systematic error. This was correctly criticized in [32], mainly because of some unavoidable consequences. The investigation to be presented here was triggered by this discussion and was aimed to improve our previous results avoiding this simplifying assumption. This has led us into a rather involved weighted Monte Carlo treatment of the collective coordinate integration, taking the Schwinger line factors completely into account in the numerically evaluated integrand. It turns out that this treatment of the second order contributions partly changes our previous conclusions.

After recalling, for the sake of completeness, the notation for field strength correlators in Section II we concentrate on the discussion of the two-instanton contribution in Section III. For the first order results we can refer to Ref. [31] where they were obtained in an analytical way. Conclusions will be drawn in Section IV.

\section{THE FIELD STRENGTH CORRELATORS IN THE SEMICLASSICAL APPROXIMATION}

The gauge invariant two-point correlators of the non-Abelian field strength are defined as

$$
\mathcal{D}_{\mu \rho, \nu \sigma}\left(x_{1}-x_{2}\right)=\left\langle 0\left|\operatorname{Tr}\left\{G_{\mu \rho}\left(x_{1}\right) S\left(x_{1}, x_{2}\right) G_{\nu \sigma}\left(x_{2}\right) S^{\dagger}\left(x_{1}, x_{2}\right)\right\}\right| 0\right\rangle,
$$


where $G_{\mu \rho}=T^{a} G_{\mu \rho}^{a}$ is the field strength tensor and $S\left(x_{1}, x_{2}\right)$ is the Schwinger-line phase factor, i.e. the parallel transporter necessary to join the field-strength operators at the points $x_{1}, x_{2}$ in order to respect gauge invariance. $T^{a}$ denotes the generators of the gauge group $S U\left(N_{c}\right)$. The most general form of the correlator compatible with Euclidean $O(4)$ rotational invariance, adequate for zero temperature, is in the notation of Ref. [1],

$$
\begin{aligned}
\mathcal{D}_{\mu \rho, \nu \sigma}(x)= & \left(\delta_{\mu \nu} \delta_{\rho \sigma}-\delta_{\mu \sigma} \delta_{\rho \nu}\right)\left(\mathcal{D}\left(x^{2}\right)+\mathcal{D}_{1}\left(x^{2}\right)\right)+ \\
& +\left(x_{\mu} x_{\nu} \delta_{\rho \sigma}-x_{\mu} x_{\sigma} \delta_{\rho \nu}+x_{\rho} x_{\sigma} \delta_{\mu \nu}-x_{\rho} x_{\nu} \delta_{\mu \sigma}\right) \frac{\partial \mathcal{D}_{1}\left(x^{2}\right)}{\partial x^{2}},
\end{aligned}
$$

with $x=x_{1}-x_{2}$ and $\mathcal{D}\left(x^{2}\right)$ and $\mathcal{D}_{1}\left(x^{2}\right)$ representing invariant vacuum structure functions. Instead of considering the invariant functions $\mathcal{D}\left(x^{2}\right)$ and $\mathcal{D}_{1}\left(x^{2}\right)$ entering Eq. (2) we shall study longitudinal and transverse correlators

$$
\begin{aligned}
& \mathcal{D}_{\|}\left(x^{2}\right)=\mathcal{D}\left(x^{2}\right)+\mathcal{D}_{1}\left(x^{2}\right)+x^{2} \frac{\partial \mathcal{D}_{1}\left(x^{2}\right)}{\partial x^{2}}, \\
& \mathcal{D}_{\perp}\left(x^{2}\right)=\mathcal{D}\left(x^{2}\right)+\mathcal{D}_{1}\left(x^{2}\right) .
\end{aligned}
$$

In particular, if $x=(0,0,0, x)$ is Euclidean timelike, we have

$$
\begin{aligned}
& \mathcal{D}_{\|}\left(x^{2}\right)=\frac{1}{3} \sum_{i} \mathcal{D}_{4 i, 4 i}\left(x^{2}\right), \\
& \mathcal{D}_{\perp}\left(x^{2}\right)=\frac{1}{3} \sum_{i<j} D_{i j, i j}\left(x^{2}\right) .
\end{aligned}
$$

It is easy to demonstrate that $\mathcal{D}_{1}$ does not contribute to the area law of Wilson loops [2, 3]. In the perturbative regime both invariant functions $\mathcal{D}$ and $\mathcal{D}_{1}$ behave like $1 / x^{4}$. Only $\mathcal{D}_{1}$ receives a contribution from one-gluon exchange. On very general grounds, the perturbative part of $\mathcal{D}$ (which appears at one loop and higher orders) was recently shown to be cancelled in the expression for the string tension by higher correlator contributions [33]. Here, we shall not discuss the perturbative contributions in more detail. Instead we will concentrate on the contribution from instantons as exclusive semiclassical configurations representing the nonperturbative part of the correlators. 
For comparison we refer to the lattice data published by the Pisa group for the two-point field-strength correlators in pure $S U(3)$ gauge theory at $T=0$ [9]. Since these data include measurements for several bare lattice couplings $\beta$, the cooling method seems to be compatible with the correct scaling behavior at high $\beta$ of the structure functions. After applying the cooling method the non-perturbative parts of the respective structure function have been fitted by an exponential function (with the same correlation length) over all distances between 0.1 and $1 \mathrm{fm}$. This contribution to $\mathcal{D}$ is considerably bigger compared to $\mathcal{D}_{1}$. This observation points towards a dominance of (anti)selfdual field entering the correlator (22). In the one-instanton approximation $\mathcal{D}_{1}$ is exactly vanishing. The structure functions extracted from the cooled Monte Carlo data still exhibit a perturbative tail $\sim x^{-4}$. In this case, however, the relative size of this contribution to $\mathcal{D}$ and $\mathcal{D}_{1}$ is not understood, in view of the above considerations.

In the lowest order (in $g^{2}$ ) semiclassical approximation for the field-strength correlator the Gaussian integral over quantum fluctuations above a single classical field configuration is separated from the gauge invariant product of field strengths to be evaluated for this background field. What then remains are zero-mode integrations over the appropriate set of collective coordinates (summarized as $\Gamma$ which characterizes the classical field) with a density function $\mathcal{M}(\Gamma)$, which results from the integration over non-zero mode fluctuations :

$$
\mathcal{D}_{\mu \rho, \nu \sigma}(x)=\frac{1}{Z} \int d \Gamma \mathcal{M}(\Gamma) \cdot \operatorname{Tr}\left\{G_{\mu \rho}\left(x_{1} ; \Gamma\right) S\left(x_{1}, x_{2} ; \Gamma\right) G_{\nu \sigma}\left(x_{2} ; \Gamma\right) S^{\dagger}\left(x_{1}, x_{2} ; \Gamma\right)\right\},
$$

where $G_{\mu \nu}(x ; \Gamma)$ is the field strength tensor and $S\left(x_{1}, x_{2} ; \Gamma\right)$ the Schwinger line corresponding to configurations $A_{\mu}(x, \Gamma)$. To be more specific, we imagine a model of the vacuum state that is semi-classically represented by superpositions of $N$ instantons and $\bar{N}$ anti-instantons [17

$$
A_{\mu}(x, \Gamma)=\sum_{i=1}^{N} A_{\mu}\left(x ; \gamma_{i}\right)+\sum_{j=1}^{\bar{N}} \bar{A}_{\mu}\left(x ; \bar{\gamma}_{j}\right)
$$

The $\gamma_{i}\left(\bar{\gamma}_{j}\right)$ denote the collective coordinates of the $i$-th instanton ( $j$-th anti-instanton), which include the positions $z_{i}$, the group space orientations $\omega_{i}$, and the sizes $\rho_{i}$. The integration measure in Eq. (5) is then expressed by

$$
d \Gamma=\prod_{i=1}^{N} d \gamma_{i} \prod_{j=1}^{\bar{N}} d \bar{\gamma}_{j}, \quad d \gamma_{i}=d^{4} z_{i} d \omega_{i} d \rho_{i}, \quad d \bar{\gamma}_{j}=d^{4} \bar{z}_{j} d \bar{\omega}_{j} d \bar{\rho}_{j} .
$$


For practical use we will consider here only instantons and antiinstantons of fixed size $\rho$. This corresponds to the instanton liquid model invented in 19 with a delta-like size distribution. A more realistic $\rho$-distribution with a selfconsistent exponential infra-red cutoff (allowing to satisfy low-energy theorems) can be obtained from the assumption that (anti-)instantons repel each other at short distances [18,20]. . .

If the instanton liquid is sufficiently dilute we can approximate the functional integral by an expansion in powers of the (anti-)instanton densities $n_{4}=N / V\left(\bar{n}_{4}=\bar{N} / V\right)$. Then it is natural first to try to neglect possible correlation effects due to interactions between instantons.

Strictly speaking, the superposition ansatz (6) makes sense as an approximate saddle point of the action only if the vector potentials $A_{\mu}, \bar{A}_{\mu}$ decrease fast enough. This happens when the singular gauge expression is used for the (anti-) instanton solutions $A_{\mu}, \bar{A}_{\mu}$, instead of the regular gauge form [17]. The existence of a systematic expansion in higher order contributions to the measure $\mathcal{M}(\Gamma)$ has been proven in Ref. [35].

In [31] the leading term in an expansion of (11) in terms of the density has been discussed in detail. In that approximation the field strength correlator is given by the sum of instanton $(I)$ and antiinstanton $(\bar{I})$ contributions

$$
\begin{aligned}
\mathcal{D}_{\mu \rho, \nu \sigma}^{(1)}\left(x_{1}, x_{2}\right)= & \mathcal{D}_{\mu \rho, \nu \sigma}^{I}+\mathcal{D}_{\mu \rho, \nu \sigma}^{\bar{I}} \\
= & n_{4} \int d^{4} z \operatorname{Tr}\left\{G_{\mu \rho}\left(x_{1} ; \gamma\right) S\left(x_{1}, x_{2} ; \gamma\right) G_{\nu \sigma}\left(x_{2} ; \gamma\right) S^{\dagger}\left(x_{1}, x_{2} ; \gamma\right)\right\} \\
& +\left(n_{4}, \gamma \rightarrow \bar{n}_{4}, \bar{\gamma}\right) .
\end{aligned}
$$

The integration over the global group orientation of the respective solution is trivial in this case and can be omitted. The Schwinger line phase factor is a path dependent matrix in the fundamental representation,

$$
S\left(x_{1}, x_{2} ; z\right)=P \exp \left(i \int_{0}^{1} d t \dot{x}_{\mu}(t) A_{\mu}(x(t) ; z)\right)
$$

*Recently, another interpretation has been put forward for such a shape as a manifestation of suppression by a Higgs like monopole condensate [34. 
where the vector potential $A_{\mu}=T^{a} A_{\mu}^{a}$, in the case at hand belongs to the single instanton source localized at $z$. Advantage has been taken from the fact that this phase factor, evaluated for a straight line has a closed expression which can be easily contracted with the field strengths.

As mentioned before, due to the vanishing of $\mathcal{D}_{1}$ for purely (anti)selfdual fields, the singleinstanton approximation reads equally for the longitudinal and transverse correlators

$$
\mathcal{D}_{\|}\left(x^{2}\right)=\mathcal{D}_{\perp}\left(x^{2}\right)=\frac{4}{3} \pi^{2} n I\left(\frac{x}{\rho}\right),
$$

where $n=n_{4}+\bar{n}_{4}$ is the total density of instantons plus antiinstantons. The function $I(x / \rho)$ (for a straight line Schwinger phase factor) is normalized by $I(0)=1$. It has been numerically computed in [31] in order to deal with the final integration over the instanton position $z$ and is plotted in Fig. 1.

\section{REVISITING THE SECOND ORDER INSTANTON DENSITY CONTRIBUTIONS TO THE FIELD STRENGTH CORRELATORS}

In this section we shall present the numerical integration giving the next order term in an expansion in terms of the instanton density. Now we have to consider the field strength from the nonlinear superposition of two different solutions $A$ and $B$, where both $A$ and $B$ can represent an instanton or antiinstanton,

$$
\begin{aligned}
G_{\mu \rho}(A, B) & =G_{\mu \rho}(A)+G_{\mu \rho}(B)+\Delta G_{\mu \rho}(A, B), \\
\Delta G_{\mu \rho}(A, B) & =-i\left\{\left[A_{\mu}, B_{\rho}\right]+\left[B_{\mu}, A_{\rho}\right]\right\} .
\end{aligned}
$$

This field strength is plugged into an integral over the (factorized) two-source weight function (with fixed sizes $\rho_{1}=\rho_{2}=\rho$ )

$$
\begin{array}{r}
\mathcal{D}_{\mu \rho, \nu \sigma}^{(2)}\left(x_{1}, x_{2}\right)=\frac{1}{2} \sum_{A, B=I, \bar{I}} n_{4}^{(A)} n_{4}^{(B)} \int d^{4} z_{1} \int d^{4} z_{2} \int d \omega_{1} \int d \omega_{2} \times \\
\operatorname{Tr}\left\{G_{\mu \rho}\left(A\left(x_{1}, \gamma_{1}\right), B\left(x_{1}, \gamma_{2}\right)\right) S\left(x_{1}, x_{2} ; \gamma_{1}, \gamma_{2}\right) G_{\nu \sigma}\left(A\left(x_{2}, \gamma_{1}\right), B\left(x_{2}, \gamma_{2}\right)\right) S^{\dagger}\left(x_{1}, x_{2} ; \gamma_{1}, \gamma_{2}\right)\right\} .
\end{array}
$$


For the purpose of our calculation, the points $x_{1}$ and $x_{2}$ are sitting on the Euclidean time axis while the two (anti)instantons are arbitrarily located in 4-d space-time. One has to keep in mind, that those formal contributions to Eq. (11), where both the factors $G_{\mu \nu}(A, B)$ at $x_{1}$ and $x_{2}$ would receive contributions from only one and the same $G_{\mu \nu}(A)$ (or $G_{\mu \nu}(B)$ ) do not really occur. They are already taken into account in the first order contribution $\mathcal{D}^{(1)}(7)$.

With the notation $y_{1}=x-z_{1}, y_{2}=x-z_{2}$ the two instanton vector potentials, both in the singular gauge, read as follows

$A_{\mu}^{a}\left(x ; z_{1}\right)=\bar{\eta}_{a \mu \nu} y_{1 \nu} f\left(y_{1}, \rho\right), B_{\mu}^{a}\left(x ; z_{2}\right)=\omega_{a a^{\prime}} \bar{\eta}_{a^{\prime} \mu \nu} y_{2 \nu} f\left(y_{2}, \rho\right), \quad f(y, \rho)=\frac{2 \rho^{2}}{y^{2}\left(y^{2}+\rho^{2}\right)}$,

where $\omega_{a a^{\prime}}$ is included to take the relative color orientation inside the pair into account. $\bar{\eta}_{a \mu \nu}$ and $\eta_{a \mu \nu}$ are the 't Hooft tensors [16]. For an antiinstanton instead of an instanton replace $\bar{\eta} \rightarrow \eta$.

The rotation of instantons in color space (the case of $S U(2)$ group)

$$
A_{\mu}^{a} \rightarrow \omega^{a b} A_{\mu}^{b}
$$

can be represented by the related rotation in $3-d$ coordinate space

$$
\begin{gathered}
\omega^{a b} A_{i}^{b}\left(x_{0}, \boldsymbol{x}\right)=\omega_{i j}^{-1} A_{j}^{a}\left(x_{0}, \omega \boldsymbol{x}\right), \\
\omega^{a b} A_{4}^{b}\left(x_{0}, \boldsymbol{x}\right)=A_{4}^{a}\left(x_{0}, \omega \boldsymbol{x}\right) .
\end{gathered}
$$

This property will be very important in the further average over the relative color orientation of two instantons.

First, let us consider the correlator $\mathcal{D}_{\|}\left(x^{2}\right)$. According to Eqs. (10,11), the evaluation of (4) naively gives rise to 16 terms. After averaging over the relative color and $3-d$ space orientations of two instantons only six of these terms give a nonzero contribution. Let us describe the contributions in some detail.

1. Two terms, for which both the $G_{4 i}\left(A\left(x_{1}, \gamma_{1}\right), B\left(x_{1}, \gamma_{2}\right)\right)$ and the $G_{4 i}\left(A\left(x_{2}, \gamma_{1}\right), B\left(x_{2}, \gamma_{2}\right)\right)$ are represented by one and the same $G_{4 i}(A)$ (or $\left.G_{4 i}(B)\right)$ do not really occur (see above). 
2. If in the place of $G_{4 i}\left(A\left(x_{1}, \gamma_{1}\right), B\left(x_{1}, \gamma_{2}\right)\right)$ the term $G_{4 i}(A)$ is taken (or $\left.G_{4 i}(B)\right)$ and in the place of $G_{4 i}\left(A\left(x_{2}, \gamma_{1}\right), B\left(x_{2}, \gamma_{2}\right)\right)$ the term $G_{4 i}(B)$ (or $G_{4 i}(A)$, respectively) the resulting contributions (2 terms) are identically zero. This can be easily seen. Under color rotation of the second instanton $B$ (or $A$, respectively) (being equivalent to a $3-d$ rotation) the term in place of $G_{4 i}\left(A\left(x_{2}, \gamma_{1}\right), B\left(x_{2}, \gamma_{2}\right)\right)$ becomes rotated, while that in place of $G_{4 i}\left(A\left(x_{1}, \gamma_{1}\right), B\left(x_{1}, \gamma_{2}\right)\right)$ does not. The rotation of the coordinates of the second instanton in the phase factor $S\left(x_{1}, x_{2} ; \gamma_{1} \gamma_{2}\right)$ etc. can be skipped, because there is an integration over these coordinates. As a result we have to average over the $3-d$ vector $G_{4 i}(B)$ (or $\left.G_{4 i}(A)\right)$ orientation leading to a vanishing contribution.

3. If in the place of $G_{4 i}\left(A\left(x_{1}, \gamma_{1}\right), B\left(x_{1}, \gamma_{2}\right)\right)$ the term $G_{4 i}(A)$ is inserted and in the place of $G_{4 i}\left(A\left(x_{2}, \gamma_{1}\right), B\left(x_{2}, \gamma_{2}\right)\right)$ the term $-i\left[A_{4}, B_{i}\right]$, the contribution is zero by the same reason as in the previous consideration. There are three other vanishing contributions to be obtained from the described one by the replacement $A \leftrightarrow B, x_{1} \leftrightarrow x_{2}$ (4 terms).

4. If in the place of $G_{4 i}\left(A\left(x_{1}, \gamma_{1}\right), B\left(x_{1}, \gamma_{2}\right)\right)$ the term $G_{4 i}(A)$ is inserted and in the place of $G_{4 i}\left(A\left(x_{2}, \gamma_{1}\right), B\left(x_{2}, \gamma_{2}\right)\right)$ the term $-i\left[B_{4}, A_{i}\right]$ the corresponding contribution is nonzero. It is independent of the color orientation of the second instanton $B$ (equivalent to its rotation in $3-d$ space) as far as the rotation of the second instanton in the phase factor $S\left(x_{1}, x_{2} ; \gamma_{1} \gamma_{2}\right)$ etc. is integrated out. There are three other analogous terms with nonzero contribution which can be obtained from the described one by the replacements $A \leftrightarrow B$, $x_{1} \leftrightarrow x_{2}$ (4 terms).

5. If in the place of $G_{4 i}\left(A\left(x_{1}, \gamma_{1}\right), B\left(x_{1}, \gamma_{2}\right)\right)$ the term $-i\left[A_{4}, B_{i}\right]$ (or $-i\left[B_{4}, A_{i}\right]$ ) is inserted and in the place of $G_{4 i}\left(A\left(x_{2}, \gamma_{1}\right), B\left(x_{2}, \gamma_{2}\right)\right)$ the term $-i\left[B_{4}, A_{i}\right]$ (or $-i\left[A_{4}, B_{i}\right]$, respectively), the contribution is vanishing by the same reason as discussed in points 2,3 above (2 terms).

6. Finally, if in the place of $G_{4 i}\left(A\left(x_{1}, \gamma_{1}\right), B\left(x_{1}, \gamma_{2}\right)\right)$ the term $-i\left[A_{4}, B_{i}\right]$ (or $\left.-i\left[B_{4}, A_{i}\right]\right)$ is inserted and in the place of $G_{4 i}\left(A\left(x_{2}, \gamma_{1}\right), B\left(x_{2}, \gamma_{2}\right)\right)$ the term $-i\left[A_{4}, B_{i}\right]$ (or $-i\left[B_{4}, A_{i}\right]$, 
respectively), the contribution is nonvanishing and independent of color rotation of the second instanton $B$ (equivalent to its rotation in $3-d$ space) by the same reason as in point 4 above (2 terms).

Now let us consider the correlator $\mathcal{D}_{\perp}\left(x^{2}\right)$. The corresponding expression in (4) naively contains 16 terms upon insertion of (10) into Eq. (11). After averaging over the relative color and $3-d$ space orientations of two instantons only four of them give nonzero contribution.

1. Two terms, for which both the $G_{i j}\left(A\left(x_{1}, \gamma_{1}\right), B\left(x_{1}, \gamma_{2}\right)\right)$ and the $G_{i j}\left(A\left(x_{2}, \gamma_{1}\right), B\left(x_{2}, \gamma_{2}\right)\right)$ are represented by one and the same $G_{i j}(A)$ (or $\left.G_{i j}(B)\right)$ do not really occur (see above).

2. If in the place of $G_{i j}\left(A\left(x_{1}, \gamma_{1}\right), B\left(x_{1}, \gamma_{2}\right)\right)$ the term $G_{i j}(A)$ is taken (or $\left.G_{i j}(B)\right)$ and in the place of $G_{i j}\left(A\left(x_{2}, \gamma_{1}\right), B\left(x_{2}, \gamma_{2}\right)\right)$ the term $G_{i j}(B)$ (or $G_{i j}(A)$, respectively) the contribution is zero. Under color rotation of the second instanton $B$ (or $A$, respectively) which is equivalent to its rotation in $3-d$ space $G_{i j}\left(A\left(x_{2}, \gamma_{1}\right), B\left(x_{2}, \gamma_{2}\right)\right)$ is rotated, while $G_{i j}\left(A\left(x_{1}, \gamma_{1}\right), B\left(x_{1}, \gamma_{2}\right)\right)$ is not. The rotation of the coordinates of the second instanton in the phase factor $S\left(x_{1}, x_{2} ; \gamma_{1} \gamma_{2}\right)$ etc. can be skipped, because of the integration over these coordinates. As a result we have the average over the orientation of a $3-d$ vector (an antisymmetric tensor is equivalent to a $3-d$ vector) $G_{i j}(B)$ (or $G_{i j}(A)$ ), which gives zero (2 terms).

3. If in the place of $G_{i j}\left(A\left(x_{1}, \gamma_{1}\right), B\left(x_{1}, \gamma_{2}\right)\right)$ the term $G_{i j}(A)$ is inserted and in the place of $G_{i j}\left(A\left(x_{2}, \gamma_{1}\right), B\left(x_{2}, \gamma_{2}\right)\right)$ the term $-i\left[A_{i}, B_{j}\right]$ the contribution is zero by the same reason as in point 2. There are three other terms with zero contribution which can be obtained from the described one by the replacements $A \leftrightarrow B, x_{1} \leftrightarrow x_{2}$ (4 terms).

4. If in the place of $G_{i j}\left(A\left(x_{1}, \gamma_{1}\right), B\left(x_{1}, \gamma_{2}\right)\right)$ the term $G_{i j}(A)$ is inserted and in the place of $G_{i j}\left(A\left(x_{2}, \gamma_{1}\right), B\left(x_{2}, \gamma_{2}\right)\right)$ the term $-i\left[B_{i}, A_{j}\right]$ the contribution is zero by the same reason as in point 2 . There are three other terms with zero contribution which can be obtained from the described one by the replacements $A \leftrightarrow B, x_{1} \leftrightarrow x_{2}$ (4 terms). 
5. If in the place of $G_{i j}\left(A\left(x_{1}, \gamma_{1}\right), B\left(x_{1}, \gamma_{2}\right)\right)$ the term $-i\left\{\left[A_{i}, B_{j}\right]+\left[B_{i}, A_{j}\right]\right\}$ (at $\left.x_{1}\right)$ is inserted and in the place of $G_{i j}\left(A\left(x_{2}, \gamma_{1}\right), B\left(x_{2}, \gamma_{2}\right)\right)$ the same term $-i\left\{\left[A_{i}, B_{j}\right]+\left[B_{i}, A_{j}\right]\right\}\left(\right.$ at $\left.x_{2}\right)$ the contribution is non-vanishing (4 terms).

With the second order contributions in terms of the instanton density included and with an additional (unknown) perturbative short-range $x^{-4}$-contribution the longitudinal and transverse correlators can be directly expressed as follows

$$
\begin{gathered}
\mathcal{D}_{\|}\left(x^{2}\right)=\frac{4}{3} \pi^{2} n I\left(\frac{x}{\rho}\right)+\frac{27}{16} \pi^{4} n^{2} \rho^{4} I_{\|}\left(\frac{x}{\rho}\right)+\frac{a_{\|}}{x^{4}}, \\
\mathcal{D}_{\perp}\left(x^{2}\right)=\frac{4}{3} \pi^{2} n I\left(\frac{x}{\rho}\right)+\frac{27}{16} \pi^{4} n^{2} \rho^{4} I_{\perp}\left(\frac{x}{\rho}\right)+\frac{a_{\perp}}{x^{4}},
\end{gathered}
$$

where $n$ is the total density of instantons and antiinstantons and $\rho$ is their size, $a_{\|}$and $a_{\perp}$ represent the coefficients of the respective perturbative contributions.

The functions $I(x / \rho), I_{\|}(x / \rho)$ and $I_{\perp}(x / \rho)$ have been obtained by numerical integration and are all normalized to 1 at $x=0$. The numerical factors come from the transition to the $S U(3)$ gauge group into which the $S U(2)$-instantons are embedded and can be calculated analytically by the consideration of correlators at $x=0$. There is a twofold nontrivial integration in $I(x / \rho)$, and a fivefold integration is implied by the expressions for $I_{\|}(x / \rho)$ and $I_{\perp}(x / \rho)$. In the last case the integrations have been carried out by means of a Monte Carlo importance sampling method. The two points $x_{1}$ and $x_{2}$ of the correlator, with $x=\left|x_{1}-x_{2}\right|$, are located on the Euclidean time axis, at times $\pm x / 2$. The two-instanton (or instanton-antiinstanton) contribution is obtained by integrating over $r_{1}, t_{1}, r_{2}, t_{2}$ and $\theta$ in a sequential manner, where the positions of the two instanton centers are $x_{1}^{I}=\left(0,0, r_{1}, t_{1}\right)$ and $x_{2}^{I}=\left(r_{2} \sin \theta, 0, r_{2} \cos \theta, t_{2}\right)$. First, variables $\left(r_{1}, t_{1}\right)$ were generated in a box of size $(20 \rho, 40 \rho)$ with a distribution proportional to $r_{1}^{2} f\left(x_{11}\right) f\left(x_{12}\right)$, where $f(x)=\frac{2}{x} \frac{\rho^{2}}{\left(x^{2}+\rho^{2}\right)}$ is the profile function of an instanton. Here, $x_{11}$ and $x_{12}$ are the $4-d$ distances of the first instanton $x_{1}^{I}$ from $x_{1}$ and $x_{2}$, respectively. For this sampling of $\left(r_{1}, t_{1}\right)$ the acceptance varied from $1 / 1000$ to $1 / 100$ for $0<x<5 \rho$. About 7000 events were accepted. Second, for each accepted event new variables $\left(r_{2}, t_{2}\right)$ were generated in a similar way, and about 100 events were accepted per each $x_{1}^{I}=\left(0,0, r_{1}, t_{1}\right)$. Finally, for each of the events accepted 
so far, an angle $\theta$ of relative orientation in $3-d$ space has been randomly selected 100 times, according to a flat measure in $\cos \theta$. The obtained accuracy of the Monte Carlo integration was about $1 \%$. The convergence of integration has been verified by doubling the integration box for $\left(r_{1}, t_{1}\right)$ and $\left(r_{2}, t_{2}\right)$ to $(40 \rho, 80 \rho)$.

The functions $I_{\|}(x / \rho)$ and $I_{\perp}(x / \rho)$ are plotted together with $I(x / \rho)$ obtained in Ref. [31] in Fig. 1. The longitudinal and transverse correlators from Eqs. (13) have been jointly fitted to the lattice data of Ref. [9] within a distance range from $0.4 \mathrm{fm}$ to $1 \mathrm{fm}$. For the parameters $n, \rho, a_{\|}$and $a_{\perp}$ we have found

$$
n=(1 \pm 0.1) \mathrm{fm}^{-4}, \quad \rho=(0.42 \pm 0.01) \mathrm{fm}, \quad \mathrm{a}_{\|}=0.46 \pm 0.02, \quad \mathrm{a}_{\perp}=0.76 \pm 0.06
$$

with an $\chi^{2} / N_{\text {dof }}=56.1 /(30-4)$. The corresponding curves for the correlations functions $\mathcal{D}_{\|}$ and $\mathcal{D}_{\perp}$ are drawn in Figs. 2 and 3, respectively, together with the lattice data. In order to give an impression how large the contribution of the different terms in Eq. (13) are, we have plotted them separately.

\section{CONCLUSIONS AND DISCUSSION}

We have considered the two-point correlators of gluon field strengths in the uncorrelated instanton liquid model up to the second order in the instanton density. The correlators have two parts: a nonperturbative one which is considered exclusively due to instantons and a perturbative one due to gluon exchange fluctuations in the vacuum. We have fitted the resulting expression directly to the lattice data within a restricted distance range. For this range the achieved quality of our fit is comparable with those of [9], where purely exponential terms

together with $x^{-4}$-contributions were fitted to the lattice data after a separation into $\mathcal{D}$ and $\mathcal{D}_{1}$. The value for the instanton density comes near to the value expected from phenomenological applications, although our present analysis describes quenched lattice data. There is a recent analysis due to A. Hasenfratz [36] which, on the basis of the two-point correlator of the topological density, gives estimates for the instanton density, the fraction of two-instanton and instanton-antiinstanton molecules, all for quenched $S U(3)$ theory and full QCD. The instanton 
density was there found to be about $1 \mathrm{fm}^{-4}$ for pure $S U(3)$, too, however the instanton size was estimated as about $0.3 \mathrm{fm}$. Thus, the mean instanton size obtained here is more in accordance with UKQCD [37]. The packing fraction of the instanton gas is estimated to $n \rho^{4} \simeq .03$ which corresponds to a reasonable diluteness.

In our fit for the range $0.4 \mathrm{fm}<\mathrm{x}<1 \mathrm{fm}$ the instanton and perturbative contributions are of a comparable order of magnitude. We obtain the relation $\mathcal{D}_{\perp}>\mathcal{D}_{\|}$now both for the perturbative and the instanton contributions. This means that both contributions to the invariant function $\mathcal{D}_{1}$ turn out to be positive. This is contrary to our previous result in [31] where we concluded that $\mathcal{D}_{1}<0$ is inavoidable within the instanton liquid description. The neglect of the Schwinger-line phase factor in the two-instanton (instanton-antiinstanton field) is to be blamed for this wrong conclusion.

The second order density terms are the final ones for transverse correlators, i.e. the $O\left(n^{3}\right), O\left(n^{4}\right), \cdots$ terms would be vanishing. For the longitudinal correlators there exist also a non-vanishing $O\left(n^{3}\right)$ contribution, but no $O\left(n^{4}\right)$ ones. The calculation of the longitudinal correlators up to this order require a nine-dimensional nontrivial integration over the threeinstanton degrees of freedom. We hope to come back to this integration in future.

\section{ACKNOWLEDGEMENTS}

The authors are grateful to S. V. Molodtsov and Yu. A. Simonov for useful discussions. The financial support through the joint RFFI-DFG project 436 RUS 113/309/10 (R) is gratefully acknowledged. 


\section{REFERENCES}

[1] H. G. Dosch, Phys. Lett. B190 (1987) 177;

H. G. Dosch and Yu. A. Simonov, Phys. Lett. B205 (1988) 339;

Yu. A. Simonov, Nucl. Phys. B324 (1989) 67.

[2] Yu. A. Simonov, Usp. Fiz. Nauk 166 (1996) 337.

[3] V. I. Shevchenko and Yu. A. Simonov, Yad Fiz. 60 (1997) 1329; transl. Phys. At. Nucl. 60 (1997) 1201.

[4] Yu. A. Simonov, S. Titard, and F. J. Yndurain, Phys. Lett. B354 (1995) 435.

[5] H. G. Dosch, E. Ferreira, and A. Krämer, Phys. Rev. D50 (1994) 1992;

O. Nachtmann, Schladming lectures 1996;

E. R. Berger and O. Nachtmann, Eur. Phys. J. C7 (1999) 459.

[6] M. Campostrini, A. Di Giacomo, and G. Mussardo, Z. Phys. C25 (1984) 173.

[7] A. Di Giacomo and H. Panagopoulos, Phys. Lett. B285 (1992) 133.

[8] L. Del Debbio, A. Di Giacomo, and Yu. A. Simonov, Phys. Lett. B332 (1994) 111.

[9] A. Di Giacomo, E. Meggiolaro, and H. Panagopoulos, e-Print Archive: hep-lat/9603017, and Nucl. Phys. Proc. Suppl. 54A (1997) 343.

[10] A. Di Giacomo, E. Meggiolaro, and H. Panagopoulos, Nucl. Phys. B483 (1997) 371.

[11] M. D’Elia, A. Di Giacomo, and E. Meggiolaro, Phys. Lett. B408 (1997) 315.

[12] G. Bali, N. Brambilla, and A. Vairo, Phys. Lett. B421 (1998) 265-272.

[13] A. Di Giacomo, Lectures given at AUTUMN 99, Lisbon, Portugal, 29 Sep - 4 Oct 1999, e-Print Archive: hep-lat/9912016.

[14] D. Antonov, e-Print Archive: hep-ph/0001193.

[15] E. Meggiolaro, Phys. Lett. B451 (1999) 414. 
[16] G.'t Hooft, Phys. Rev. D14 (1976) 3432.

[17] C. G. Callan, R. Dashen, and D. J. Gross, Phys. Rev. D17 (1978) 2717.

[18] E.-M. Ilgenfritz and M. Müller-Preussker, Nucl. Phys. B184 (1981) 443.

[19] E. V. Shuryak, Nucl. Phys. B203 (1982) 93, 116, 140; Nucl. Phys. B328 (1989) 85, 102.

[20] D. I. Diakonov and V. Yu. Petrov, Nucl. Phys. B245 (1984) 259;

D. I. Diakonov, V. Yu. Petrov, and P. V. Pobylitsa, Phys. Lett. B226 (1989) 471.

[21] D. I. Diakonov, Talk given at Varenna 1995, e-Print Archive: hep-ph/9602375.

[22] T. Schäfer and E. V. Shuryak, Rev. Mod. Phys. 70 (1998) 323-426.

[23] J. W. Negele, Nucl. Phys. Proc. Suppl. 73 (1999) 92.

[24] M. Teper, Plenary talk LATTICE 99, Pisa, Italy, 29 Jun - 3 Jul 1999. e-Print Archive: hep-lat/9909124.

[25] M. Fukushima, H Suganuma, and H. Toki, Phys. Rev. D60 (1999) 094504.

[26] D. Chen, R. C. Brower, J. W. Negele, and E. V. Shuryak, Nucl. Phys. Proc. Suppl. 73 (1999) 512 .

[27] E.-M. Ilgenfritz and S. Thurner, e-Print Archive: hep-lat/9810010.

[28] M. Fukushima, S. Sasaki, H. Suganuma, A. Tanaka, H. Toki, and D. Diakonov, Phys. Lett. B399 (1997) 141.

[29] V. N. Baier and Yu. F. Pinelis, Phys. Lett. B116 (1982) 179.

[30] A. E. Dorokhov, S. V. Esaibegyan, and S. V. Mikhailov, Phys. Rev. D56 (1997) 4062.

[31] E.-M. Ilgenfritz, B. V. Martemyanov , S. V. Molodtsov, M. Müller-Preussker, and Yu. A. Simonov , Phys. Rev. D58 (1998) 114508.

[32] A. E. Dorokhov, S. V. Esaibegyan, A. E. Maximov, and S. V. Mikhailov, e-Print Archive: 
hep-ph/9903450.

[33] V. I. Shevchenko and Yu. A. Simonov, Phys. Lett. B437 (1998) 146-152.

[34] E. V. Shuryak, e-Print Archive: hep-ph/9909458.

[35] H. Levine and L. G. Yaffe, Phys. Rev. D19 (1979) 1225.

[36] A. Hasenfratz, e-Print Archive: hep-lat/9912053.

[37] D. A. Smith and M. J. Teper, Phys. Rev. D58 (1998) 014505. 


\section{FIGURES}

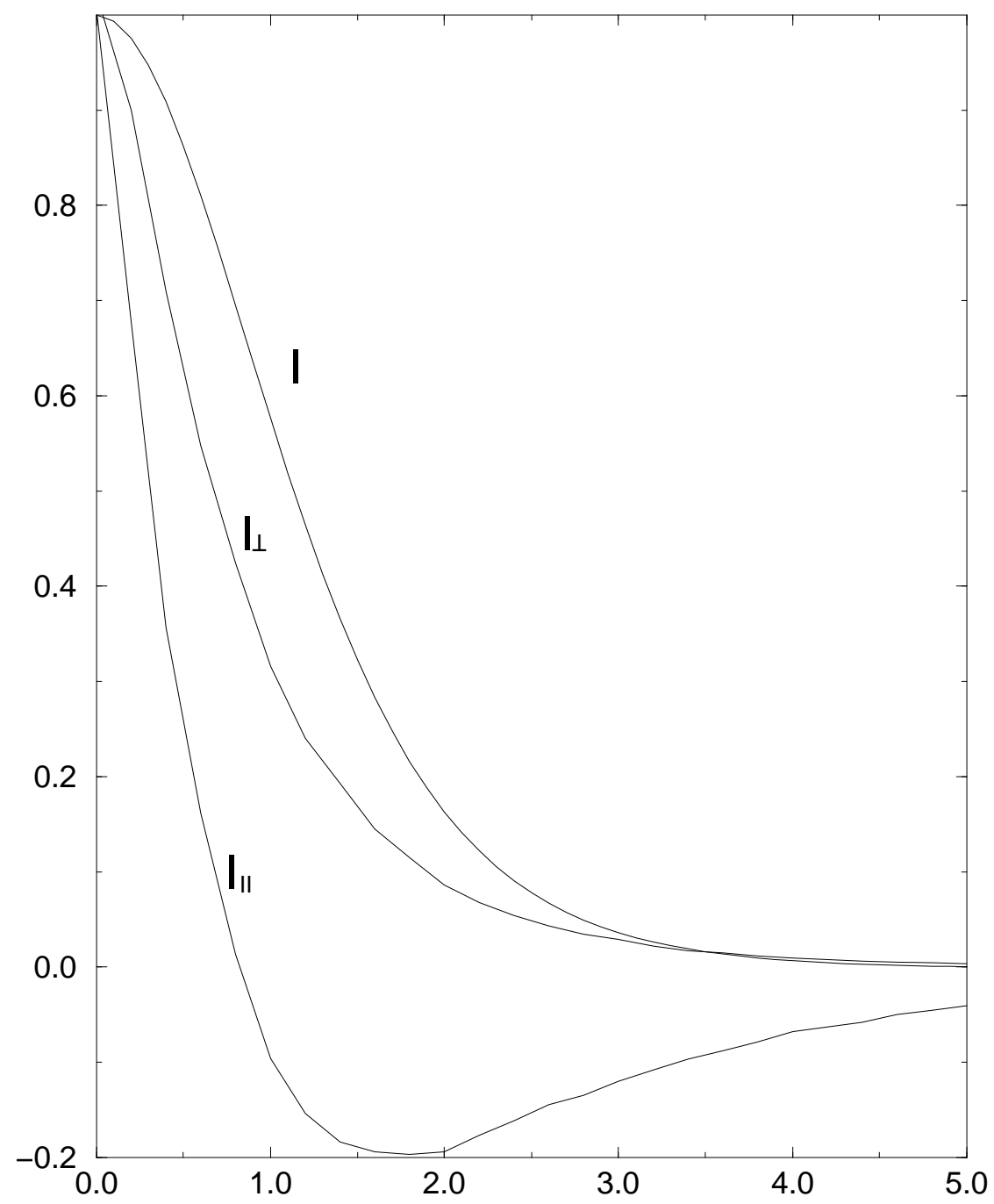

FIG. 1. The numerically computed functions $I(x / \rho)$ for the first order instanton contribution (from [31]) and $I_{\|}(x / \rho), I_{\perp}(x / \rho)$ for the second order contribution. 


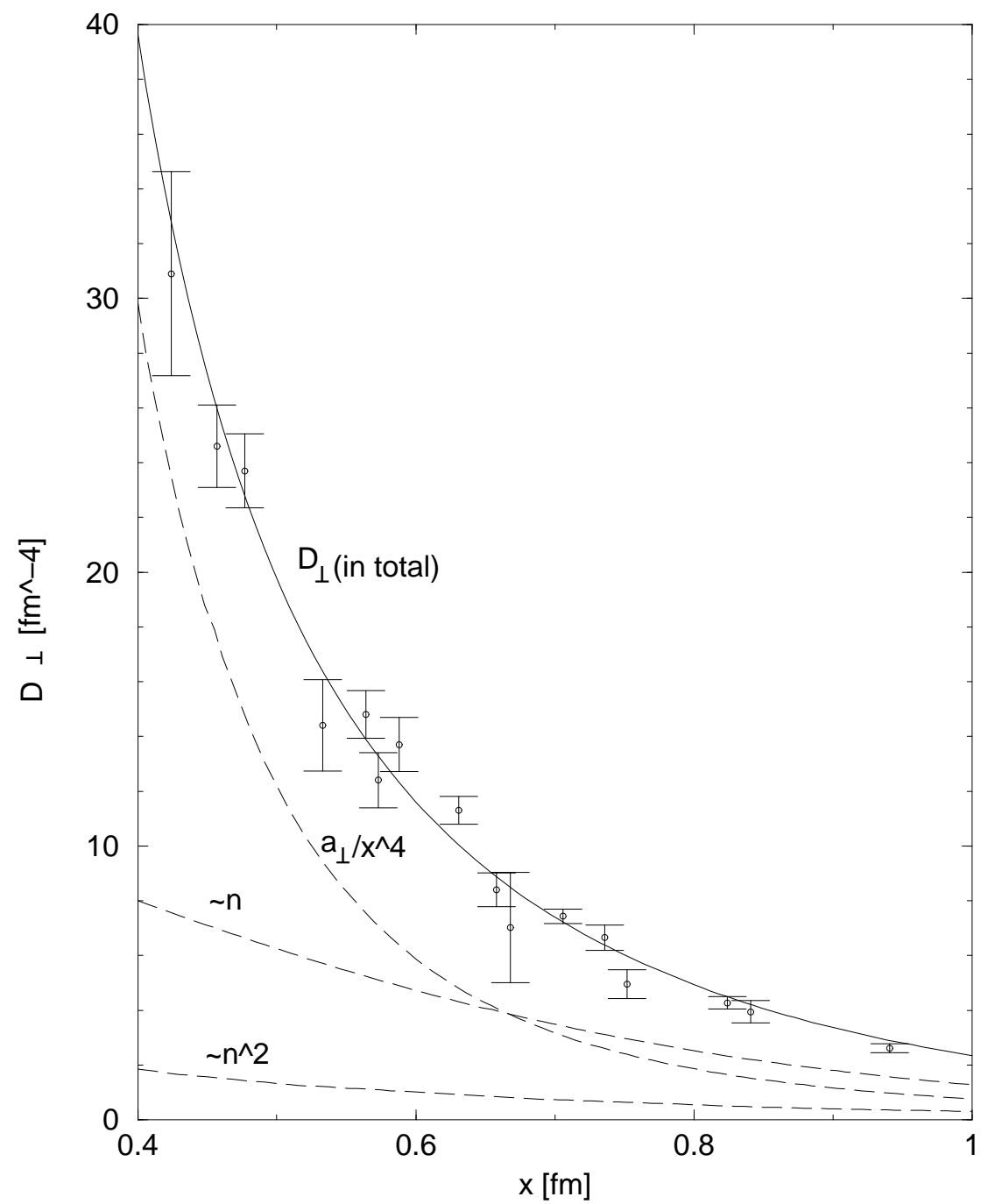

FIG. 2. Transverse field strength correlator as described by the instanton gas model (continuous line) and fitted to the lattice data points of Ref. [9] The dashed lines show separately the first and second order instanton density contributions as well as the perturbative $x^{-4}$ contribution. 


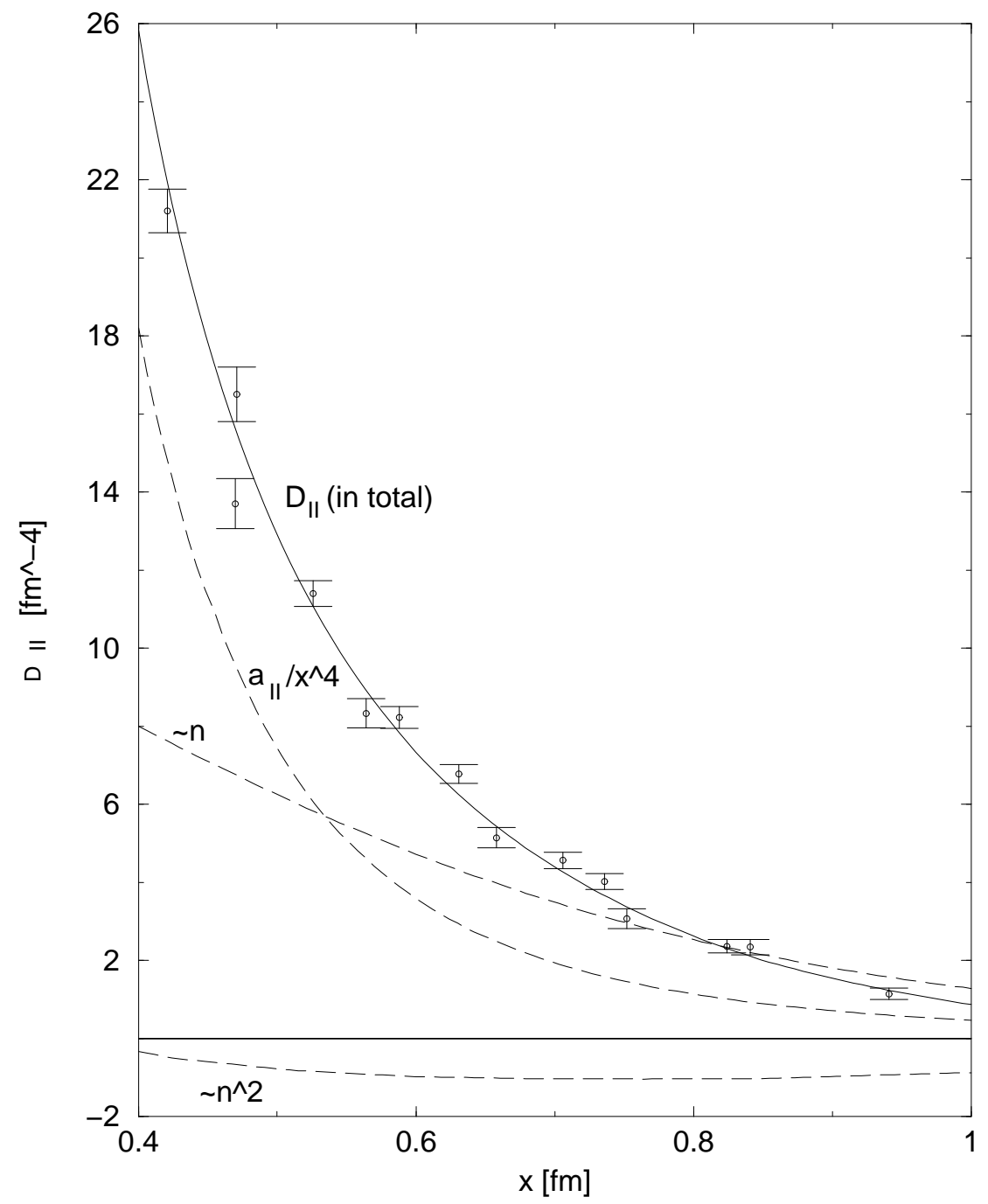

FIG. 3. Same as in Figure IV, but for the longitudinal field strength correlator. 